\title{
Lung Transplantation and Survival in Children with Cystic Fibrosis
}

\author{
Theodore G. Liou, M.D., Frederick R. Adler, Ph.D., David R. Cox, Ph.D., and Barbara C. Cahill, \\ M.D. \\ From the Departments of Internal Medicine (T.G.L., B.C.C.), Mathematics (F.R.A.), Biology (F.R.A.), \\ and Pediatrics (T.G.L.), and the Intermountain Cystic Fibrosis Center (T.G.L.) and the Lung \\ Transplant Program (B.C.C.), University of Utah, Salt Lake City; and Nuffield College, Oxford, United \\ Kingdom (D.R.C.)
}

\begin{abstract}
BACKGROUND-The effects of lung transplantation on the survival and quality of life in children with cystic fibrosis are uncertain.

METHODS-We used data from the U.S. Cystic Fibrosis Foundation Patient Registry and from the Organ Procurement and Transplantation Network to identify children with cystic fibrosis who were on the waiting list for lung transplantation during the period from 1992 through 2002. We performed proportional-hazards survival modeling, using multiple clinically relevant covariates that were available before the children were on the waiting list and the interactions of these covariates with lung transplantation as a time-dependent covariate. The data were insufficient in quality and quantity for a retrospective quality-of-life analysis.
\end{abstract}

RESULTS-A total of 248 of the 514 children on the waiting list underwent lung transplantation in the United States during the period from 1992 through 2002. Proportional-hazards modeling identified four variables besides transplantation that were associated with changes in survival. Burkholderia cepacia infection decreased survival, regardless of whether the patient underwent transplantation. A diagnosis of diabetes before the patient was placed on the waiting list decreased survival while the patient was on the waiting list but did not decrease survival after transplantation, whereas older age did not affect waiting-list survival but decreased post-transplantation survival. Staphylococcus aureus infection increased waiting-list survival but decreased post-transplantation survival. Using age, diabetes status, and S. aureus infection status as covariates, we estimated the effect of transplantation on survival for each patient group, expressed as a hazard factor of less than 1 for a benefit and more than 1 for a risk of harm. Five patients had a significant estimated benefit, 315 patients had a significant risk of harm, 76 patients had an insignificant benefit, and 118 patients had an insignificant risk of harm associated with lung transplantation.

CONCLUSIONS-Our analyses estimated clearly improved survival for only 5 of 514 patients on the waiting list for lung transplantation. Prolongation of life by means of lung transplantation should not be expected in children with cystic fibrosis. A prospective, randomized trial is needed to clarify whether and when patients derive a survival and quality-of-life benefit from lung transplantation.

End-stage lung disease causes about $80 \%$ of all deaths among patients with cys tic fibrosis. 1 The median age at death is approximately 25 years. ${ }^{1}$ Every year, many children with cystic fibrosis die from respiratory failure.

Address reprint requests to Dr. Liou at the Division of Respiratory, Critical Care, and Occupational Pulmonary Medicine, 26 N. Medical Dr., Salt Lake City, UT 84132, or at ted.liou@utah.edu. 
Lung transplantation is the most aggressive therapy available for end-stage lung disease, and cystic fibrosis is the most frequent indication for lung transplantation in children. ${ }^{2}$ This highrisk procedure is costly, and the associated effect on the patient's quality of life is uncertain. Complications associated with transplantation account for $12 \%$ of all deaths among patients with cystic fibrosis, making transplantation the second leading cause of death after end-stage lung disease. ${ }^{1}$

Several retrospective studies have evaluated the survival benefit of lung transplantation for patients with cystic fibrosis. ${ }^{3-7}$ An analysis of data from 124 children with cystic fibrosis referred for lung transplantation, including 47 children who underwent the procedure at the Great Ormond Street Hospital in the United Kingdom during the period from 1988 through 1998, showed improved survival. ${ }^{6}$ More recently, our analysis of data from 205 children who underwent transplantation and 1018 children who did not undergo transplantation from the United States showed no survival benefit. 7

Possible explanations for the discrepancy between these findings include differences in patient characteristics, patient-selection policies, and transplantation and analytic methods. ${ }^{8,9}$ To address some of the issues, we analyzed a large data set of children with cystic fibrosis who underwent lung transplantation. We used proportional-hazards modeling with time-dependent covariates to consider the effects of multiple covariates before and after transplantation. $10-13$

\section{METHODS}

\section{PATIENTS}

We used data from the Cystic Fibrosis Foundation Patient Registry (CFFPR), which includes longitudinal information on patients from 117 certified cystic fibrosis centers during the period from 1992 through 2002. We also used data from the Organ Procurement and Transplantation Network (OPTN) from 1988 through 2004. The OPTN data include information on all patients ever placed on the waiting list for lung transplantation in the United States. We matched and verified patients between these data sets using patient-specific identifiers, and we examined each match for confirmation.

Our project was reviewed and approved by the investigational review board of the University of Utah, the Cystic Fibrosis Foundation, and the OPTN. Informed consent specific to this study was not required.

\section{STUDY DESIGN}

Because children who underwent lung transplantation were previously on the waiting list, we could not directly compare the survival of patients on the waiting list with the survival of patients after transplantation using, for example, Kaplan-Meier statistics. However, with the use of proportional-hazards methods with transplantation as a covariate that changed at the time of transplantation, we could estimate how the procedure altered the risk of death. 10,12 The modeling of transplantation as a time-dependent covariate was introduced in 1977 to analyze the effects of heart transplantation on survival. ${ }^{11}$ In the present study, we analyzed lung transplantation as a time-dependent covariate. We derived hazard factors that reveal the associated multiplicative change in the risk of death due to lung transplantation. Detailed modeling procedures are described in the Supplementary Appendix, available with the full text of this article at www.nejm.org.

\section{STATISTICAL ANALYSIS}

Using three different methods, we tested for a departure from the proportional-hazards assumption that the effect of an explanatory variable on the hazard is constant in time. ${ }^{14}$ First, 
with the use of S-plus 7.0 software (Insightful), we applied the S-plus procedure called cox.zph to the final model. Second, we separated study patients into two roughly equal groups according to the year of their placement on the waiting list (during the period from 1992 through 1998 or from 1999 through 2002), and we compared the resulting proportional-hazards models with each other and with the final model. Finally, we tested for the effects of higher mortality immediately after transplantation ${ }^{3}$ by introducing a time-dependent covariate ${ }^{10}$ for survival beyond the first 6 months after transplantation.

We performed two additional tests of model stability. We examined the effect of loss to followup ${ }^{15}$ in our final model (see the Supplementary Appendix), and we applied bootstrapping techniques on our final model to rule out the possibility that a small number of patients with unusual characteristics accounted for the results of our analysis. ${ }^{16}$

We examined potential markers of quality of life, including days of hospitalization and complications of disease per year. For this analysis, we compared values in the year before transplantation with those 1 and 2 years after transplantation among surviving patients.

\section{RESULTS}

PATIENTS

The CFFPR for the period from 1992 through 2002 contains data on 31,394 patients with cystic fibrosis. The OPTN has data for 21,679 patients who were on the waiting list during the period from 1988 through 2004. We identified 3364 patients with cystic fibrosis who were placed on the lung-transplantation waiting list during the period from 1992 through 2002; 602 of these patients were younger than 18 years of age. We excluded 10 patients for whom lung-function data were not available, 2 patients with missing microbiologic data, 4 patients with missing data regarding acute exacerbations, 2 patients with recorded death dates that preceded their placement on the waiting list, and 70 patients with missing data during the 2 years before placement on the waiting list. After these patients had been excluded, there were 514 patients, or $85 \%$ of all children with cystic fibrosis listed for transplantation in the United States during the study period. The median survival for patients who died before transplantation was 223 days. The median time to transplantation for the 248 patients who underwent transplantation was 427 days. The median survival after transplantation was 1037 days (Fig. 1).

\section{DEATHS}

A total of 141 patients who were on the waiting list died, and 120 patients died after undergoing transplantation (Table 1). Respiratory failure caused $91 \%$ of the deaths among patients who were on the waiting list. After transplantation, $60 \%$ of deaths were due to complications of transplantation (primarily allograft rejection), $29 \%$ of deaths were due to respiratory failure, and $11 \%$ of deaths were due to other causes.

\section{PROPORTIONAL-HAZARDS MODELING}

We began to develop our model with 26 covariates and their interaction terms with transplantation (see Table A in the Supplementary Appendix). Backward selection resulted in a final model with four covariates besides lung transplantation and three interaction terms. Tests of the proportional-hazards assumption did not show a departure from proportionality. $10,12,14$ Results were not affected by the method of determining loss to follow-up for the 85 patients on the waiting list who did not undergo transplantation before the end of the study and the 128 patients who were alive at the end of the study. The coefficients and calculated hazard factors were shown to be robust with bootstrapping. 16 
To facilitate interpretation, we recoded interaction terms to isolate effects before and after transplantation, thus revealing how covariate effects changed with this procedure (Table 2). 12,13 Older age at study entry was associated with improved survival before transplantation but decreased survival after transplantation. Diabetes in patients before study entry was associated with reduced survival before transplantation but was not significantly associated with survival after transplantation. Staphylococcus aureus infection was associated with improved survival before transplantation but with greatly reduced survival afterward. Burkholderia cepacia infection did not modify the effect of transplantation, but it was associated with decreased survival for all affected patients.

Forward-selection procedures were used to reconsider the effects of lung function expressed as the percent of the predicted value for forced expiratory volume in 1 second $\left(\mathrm{FEV}_{1} \%\right)$ and infections with Achromobacter xylosoxidans, methicillin-resistant $S$. aureus, Stenotrophomonas maltophilia, Pseudomonas aeruginosa, mucoid $P$. aeruginosa, and other pseudomonas species. None of the results were significant. Using the calendar date of transplantation, we found some suggestion, short of statistical significance, that survival prospects decreased in the later years of the study (see Table B in the Supplementary Appendix). To determine specifically whether the effects of the patient's age on the outcome of transplantation persist into adulthood, we applied the final candidate model to the 2744 adults with cystic fibrosis whom we identified. We found no interaction of age with transplantation (see Table C in the Supplementary Appendix).

Values for the partial pressure of carbon dioxide in arterial blood $\left(\mathrm{PaCO}_{2}\right)$ were available for only 299 of the 514 children studied; thus, this covariate was excluded from the main analysis in order to preserve statistical power and avoid bias. In a subgroup analysis, $\mathrm{PaCO}_{2}$ did not have a significant effect on survival. Similarly, results of the patient's 6-minute walk test and serum creatinine level and hospitalization status had no effect. Dependence on supplemental oxygen seemed to decrease survival. However, oxygen use was inconsistently recorded when we compared CFFPR and OPTN data, so that the magnitude and significance of the result were uncertain. Poorer functional status was associated with decreased survival among patients for whom data on functional status were available but did not have an interaction with transplantation. Data on pulmonary-artery pressure were sparse, and the number of patients requiring mechanical ventilation at the time of placement on the waiting list was too small to perform a meaningful analysis.

\section{MODEL INTERPRETATION}

Recoding covariates with interactions 12,13 improves an understanding of covariate effects, but understanding the clinical effect of lung transplantation requires adaptation of the model as far as feasible to individual circumstances. ${ }^{8}$ Calculation of transplantation hazard factors according to age, diabetes status, and S. aureus infection status at the time of placement on the waiting list for each of the 514 children in the study showed hazard factors in four categories: significant estimated benefit (5 patients), significant risk of harm (315 patients), insignificant benefit (76 patients), and insignificant risk of harm (118 patients) (Fig. 2A). The distribution of estimated effects was similar for the 248 patients who underwent transplantation: 1 patient for whom transplantation was estimated to be beneficial, 162 for whom it was estimated to be harmful, and 85 for whom benefit was indeterminate (Fig. 2B).

Because our patients had a wide range of lung function and prognoses, we examined results for patients categorized according to $\mathrm{FEV}_{1} \% 17$ and 5-year predicted survival. $5,7 \mathrm{We}$ found no threshold of $\mathrm{FEV}_{1} \%$ or 5 -year predicted survival below which the hazard factor for death associated with transplantation was generally reduced (Fig. 3). 


\section{QUALITY OF LIFE}

We examined the data for changes in the number of days of hospitalization, number of complications, and incidence of bronchiolitis obliterans. After transplantation, the number of days of hospitalization appeared to decrease, but complications appeared to increase, resulting in uncertain implications for quality of life (see Fig. A in the Supplementary Appendix). Reporting biases, especially lack of reporting of bronchiolitis obliterans, further reduced the applicability of these findings (see the Supplementary Appendix).

\section{DISCUSSION}

This retrospective study of children with cystic fibrosis who were selected for lung transplantation showed that most of the children (509 of the 514 studied and 247 of the 248 patients who underwent transplantation) did not derive a significant estimated survival benefit. Although the majority of patients (315) were at significant risk for harm, for 194 patients, the procedure was not clearly harmful or beneficial. Less than $1 \%$ of patients had a significant estimated survival benefit (Fig. 2). In contrast to earlier studies examining primarily adults with cystic fibrosis, ${ }^{5,7}$ there was no lower threshold of 5 -year predicted survival ${ }^{4}$ below which children with cystic fibrosis had an estimated survival benefit due to lung transplantation. Similarly, there was no such lower threshold for $\mathrm{FEV}_{1} \%$ (Fig. 3).

Our results reflect essentially the entire U.S. experience with pediatric lung transplantation for cystic fibrosis during the period from 1992 through 2002. Actuarial survival for lung transplantation for cystic fibrosis has not appreciably changed in the past several years 2 ; thus, the ability of our model to predict survival outcomes for patients undergoing transplantation after 2002 is likely to be high.

On the basis of results of proportional-hazards modeling (Table 2), children with cystic fibrosis could be categorized into four clinically identifiable groups that varied according to the outcome of lung transplantation (Fig. 2). Within each group, outcomes of transplantation worsened with increasing age, but the effect of age disappeared for patients who were 18 years of age or older (see Table C in the Supplementary Appendix).

Children who were infected with S. aureus and children who were 12 years of age or older were highly likely to be harmed, whereas younger children with $S$. aureus infection had no clear benefit or risk of harm from transplantation. The results (Table 2) confirmed previous results that showed that $S$. aureus infection improves survival among patients with cystic fibrosis who have not undergone transplantation. ${ }^{4}$ The protective effect of $S$. aureus infection is most likely due to active competition between $S$. aureus and more harmful $P$. aeruginosa organisms in the airways of patients with cystic fibrosis (unpublished data). After transplantation, S. aureus infection was nearly as hazardous as B. cepacia infection (Table 2), perhaps as a side effect of immunosuppression.

The two youngest patients with diabetes may have had a significant survival benefit with transplantation, whereas the remaining patients had no clear change in survival. The absence of high hazard factors suggesting harm may reflect the tendency for diabetes to develop in these patients after transplantation. In essence, patients with diabetes have already paid some of the cost of transplantation and thus are less likely to be harmed (Fig. 2A). Of the 15 children with both $S$. aureus infection and diabetes, none had a significant benefit from transplantation, but 2 had a significant risk of harm.

Among 318 children with neither S. aureus infection nor diabetes, 192 were at risk for decreased survival, 3 appeared to have had a significant survival benefit, and the remainder had an uncertain effect of lung transplantation (see Table D in the Supplementary Appendix). 
However, the three patients with a survival benefit were at the extreme younger end of the age spectrum, where there were relatively fewer data, raising the concern that the estimated benefit results from the overextrapolation of data rather than a real reduction in hazard (Fig. 2A).

Many studies have analyzed the survival effects of multiple covariates besides lung transplantation. $3,5-7,11,18,19$ However, this study identifies covariates that create different effects depending on transplantation status, which is the key issue for determining the appropriateness of transplantation. In the presence of such effects, individual characteristics determine the effect of transplantation, and it becomes possible to estimate the effect of transplantation for precise clinical circumstances (Fig. 2) instead of having to rely on a single generalized estimate (see Table E in the Supplementary Appendix). ${ }^{3,6}$

Our study of children with cystic fibrosis most closely resembles the study by Aurora et al. ${ }^{6}$ but our results suggesting that a survival benefit of lung transplantation is unlikely are seriously discrepant. Reconciling the discrepancy requires consideration of differences in analytic methods, methods of calculating survival after transplantation, characteristics of the patients studied, and waiting-list management. However, this study involved four times as many patients and transplant recipients as those in the study by Aurora et al.; this affords our study much more statistical power (see the Supplementary Appendix).

The retrospective nature of our study raises the possibility of biases in patient selection for transplantation. The proportional-hazards model corrects for these biases, provided that there are no unobserved patient characteristics with a substantial effect both on the decision to undergo transplantation and on survival. If, for example, healthier patients generally waited longer for transplantation, the results would appear to be biased against transplantation.

Our analysis involved children with cystic fibrosis who were already selected for lung transplantation, a subgroup that may not be representative of patients with cystic fibrosis and severe lung disease. In the United States, patients are selected to undergo transplantation because of low lung function, increasing numbers of exacerbations, and other factors that may indicate a poor prognosis. ${ }^{17,20,21}$ The use of these specific factors to choose patients may explain the absence of $\mathrm{FEV}_{1} \%$ and weight from the model (Table 2) as well as the absence of a significant association between $\mathrm{PaCO}_{2}$ and outcomes. Thus, our ability to derive selection criteria for transplantation with broad applicability among children with cystic fibrosis might have been limited. Nevertheless, the finding that only 1 of 248 children with cystic fibrosis who underwent transplantation during the period from 1992 through 2002 had clearly improved survival (Fig. 2B) suggests that the factors used to select candidates for transplantation could not identify patients who were likely to have a survival benefit.

Our model has little similarity to the lung allocation score for prioritizing U.S. candidates for lung transplantation. ${ }^{22}$ This score determines the order of transplantation for patients on the waiting list with several dissimilar end-stage lung diseases, whereas our study focused on selecting patients with cystic fibrosis for transplantation. We analyzed key variables in the CFFPR and data from patients younger than 12 years of age that were unavailable or excluded from the development of the lung allocation score. The OPTN will revise this scoring system on the basis of accrued experience to improve management of the waiting list. However, management of the list can be improved more effectively if there is improved initial patient selection, the goal of this study.

We cannot comment on the effect of lung transplantation on the quality of life for children with cystic fibrosis that is so severe that they are considered for this procedure. Two prospective, cross-sectional studies of quality of life, involving 280 transplant recipients (61 with cystic fibrosis) and 108 transplant recipients (10 with cystic fibrosis), showed quality-of-life improvements for survivors. ${ }^{23,24}$ However, the benefits varied widely and were countered by 
increasing illnesses, especially bronchiolitis obliterans, a common form of allograft rejection, and by decreased survival. Our analysis of indirect indicators of quality of life produced mixed results of uncertain quality (see the Supplementary Appendix).

The results underscore that sustained, multi-disciplinary care rather than lung transplantation is central to longevity in children with cystic fibrosis. Comprehensive, evidence-based care $^{25}$ maximizes survival and quality of life by preserving health and avoiding transplantation. Lung transplantation in adulthood, if needed, can be undertaken with a greater probability of increased survival. ${ }^{7}$

Our results suggest several choices: continuation of lung transplantation in children with cystic fibrosis for potential improvement in quality of life, discontinuation of transplantation to avoid decreased survival, or consideration of a prospective, randomized trial of lung transplantation for children with cystic fibrosis, combined with an assessment of quality of life. The first option entails permitting well-informed persons ${ }^{26}$ to seek uncertain quality-of-life improvement despite the likelihood that lung transplantation will cause decreased survival. The second option rules out the potential benefit for the sizable group of patients for whom our study could not estimate a significant survival benefit or risk of harm. The last option requires overcoming daunting logistical challenges and challenges in trial design, especially if quality of life is included as an end point along with survival. ${ }^{26}$ Despite the challenges, we believe that the burden of proof has shifted: a beneficial effect of lung transplantation can no longer be assumed, 19 and a study is needed to show a survival or quality-of-life benefit for a well-defined group of children with cystic fibrosis. Only a prospective trial can fully address potential biases, search for additional selection criteria, thoroughly evaluate the effect of transplantation on quality of life, and show the effect of the procedure on survival.

\section{Supplementary Material}

Refer to Web version on PubMed Central for supplementary material.

\section{Acknowledgements}

Supported in part by a Harry Shwachman Clinical Investigator Award, a clinical research grant from the U.S. Cystic Fibrosis Foundation, the Modeling the Dynamics of Life Fund at the University of Utah, and a grant from the Ben B. and Iris M. Margolis Foundation of Utah.

Dr. Liou reports serving as a medical and statistical consultant for the Epidemiologic Study of Cystic Fibrosis for Genentech (South San Francisco, CA), for the Registry to Evaluate Early and Long-term Pulmonary Arterial Hypertension Disease Management for Actelion (Allschwil, Switzerland), for the Project on Adult Care in Cystic Fibrosis for the Educational Development Center (Newton, MA), and for the Gehrson Lehman Council of Advisors (Austin, TX), as a clinical site coinvestigator for phase 2 and 3 studies of inhaled aztreonam for Gilead Sciences (Foster City, CA), inhaled denufosol for Inspire Pharmaceuticals (Durham, NC), pancreatic enzymes for Axcan Pharma (Montreal), Altus Pharmaceuticals (Cambridge, MA), and Eurand (Milan), tiotropium for Boehringer Ingelheim (Ingelheim, Germany), and MPEX-376, a new inhaled agent, for Mpex Pharmaceuticals (San Diego, CA), and serving on the CFFPR committee of the U.S. Cystic Fibrosis Foundation. Drs. Liou and Cahill report having served on the Lung Review Board for the United Network for Organ Sharing and the OPTN. No other potential conflict of interest relevant to this article was reported.

Some of the data reported here were supplied by the United Network for Organ Sharing as the contractor for the OPTN. The interpretation and reporting of these data are the responsibility of the authors and should not be seen as an official policy of or interpretation by the OPTN or the U.S. government. The remaining data reported were supplied by the U.S. Cystic Fibrosis Foundation. The authors are responsible for interpretation and reporting of these data and do not express opinions or recommendations for the foundation.

We thank the OPTN and the Cystic Fibrosis Foundation for access to data and Drs. Greg Elliott, John Michael, Holly Carveth, Boaz Markewitz, Tom Kennedy, John Hoidal, David Huang, Amrapali Shah, Robert Paine, and Tom Keens for critical appraisals of an earlier version of the manuscript. Dr. Liou thanks Dr. Marlyn Woo, Evelynn Hsu, and Lynn Fukushima, R.N., for their assistance. 


\section{References}

1. Cystic Fibrosis Foundation Patient Registry. 2002 annual data report to the center directors. Bethesda, MD: Cystic Fibrosis Foundation; 2003.

2. Department of Health and Human Services. Annual report of the U.S. Organ Procurement and Transplantation Network and the Scientific Registry of Transplant Recipients: transplant data 19952004. Rockville, MD: Health Resources and Services Administration, Healthcare Systems Bureau, Division of Transplantation; 2005.

3. Hosenpud JD, Bennett LE, Keck BM, Edwards EB, Novick RJ. Effect of diagnosis on survival benefit of lung transplantation for end-stage lung disease. Lancet 1998;351:24-7. [PubMed: 9433425]

4. Liou TG, Adler FR, Fitzsimmons SC, Cahill BC, Hibbs JR, Marshall BC. Predictive 5-year survivorship model of cystic fibrosis. Am J Epidemiol 2001;153:345-52. [PubMed: 11207152]

5. Liou TG, Adler FR, Cahill BC, et al. Survival effect of lung transplantation for patients with cystic fibrosis. JAMA 2001;286:2683-9. [PubMed: 11730443]

6. Aurora P, Whitehead B, Wade A, et al. Lung transplantation and life extension in children with cystic fibrosis. Lancet 1999;354:1591-3. [PubMed: 10560673]

7. Liou TG, Adler FR, Huang D. Use of lung transplantation survival models to refine patient selection in cystic fibrosis. Am J Respir Crit Care Med 2005;171:1053-9. [PubMed: 15695493]

8. Sweet, SC.; Faro, A., editors. Am J Respir Crit Care Med. 173. 2006. International Pediatric Lung Transplant Collaborative. Not so fast - don't deprive children with cystic fibrosis of the option for lung transplantation; p. 246-7.

9. Liou TG, Adler FR, Huang D. Not so fast - don't deprive children with cystic fibrosis of the option for lung transplantation. Am J Respir Crit Care Med 2006;173:247-8.

10. Cox DR. Regression models and life-tables. J R Stat Soc [B] 1972;34:187-220.

11. Crowley J, Hu M. Covariance analysis of heart transplant survival data. J Am Stat Assoc 1977;72:2736.

12. Cox, DR.; Oakes, D. Analysis of survival data. London: Chapman \& Hall; 1984.

13. Therneau, TM.; Grambsch, PM. Modeling survival data: extending the Cox model. New York: Springer-Verlag; 2000.

14. Grambsch PM, Therneau TM. Proportional hazards tests and diagnostics based on weighted residuals. Biometrika 1994;81:515-26.

15. Britton A, Murray D, Bulstrode C, McPherson K, Denham R. Loss to followup: does it matter. Lancet 1995;345:1511-2. [PubMed: 7769925]

16. Davison, AC.; Hinkley, DV. Bootstrap methods and their application. New York: Cambridge University Press; 1997.

17. Kerem E, Reisman J, Corey M, Canny GJ, Levison H. Prediction of mortality in patients with cystic fibrosis. N Engl J Med 1992;326:1187-91. [PubMed: 1285737]

18. Sharples L, Hathaway T, Dennis C, Caine N, Higenbottam T, Wallwork J. Prognosis of patients with cystic fibrosis awaiting heart and lung transplantation. J Heart Lung Transplant 1993;12:669-74. [PubMed: 8369328]

19. Geertsma A, Ten Vergert EM, Bonsel GJ, de Boer WJ, van der Bij W. Does lung transplantation prolong life? A comparison of survival with and without transplantation. J Heart Lung Transplant 1998;17:511-6. [PubMed: 9628571]

20. Yankaskas JR, Mallory GB Jr. Lung transplantation in cystic fibrosis: consensus conference statement. Chest 1998;113:217-26. [PubMed: 9440593]

21. Maurer JR, Frost AE, Glanville AR, et al. International guidelines for the selection of lung transplant candidates. Am J Respir Crit Care Med 1998;158:335-9. [PubMed: 9655748]

22. Egan TM, Murray S, Bustami RT, et al. Development of the new lung allocation system in the United States. Am J Transplant 2006;6:1212-27. [PubMed: 16613597]

23. Smeritschnig B, Jaksch P, Kocher A, et al. Quality of life after lung transplantation: a cross-sectional study. J Heart Lung Transplant 2005;24:474-80. [PubMed: 15797751]

24. Kugler C, Fischer S, Gottlieb J, et al. Health-related quality of life in two hundred-eighty lung transplant recipients. J Heart Lung Transplant 2005;24:2262-8. [PubMed: 16364880] 
25. Gibson RL, Burns JL, Ramsey BW. Pathophysiology and management of pulmonary infections in cystic fibrosis. Am J Respir Crit Care Med 2003;168:918-51. [PubMed: 14555458]

26. Cox DR, Fitzpatrick R, Fletcher AE, et al. Quality-of-Life assessment: can we keep it simple? J R Stat Soc [A] 1992;155:353-93. 


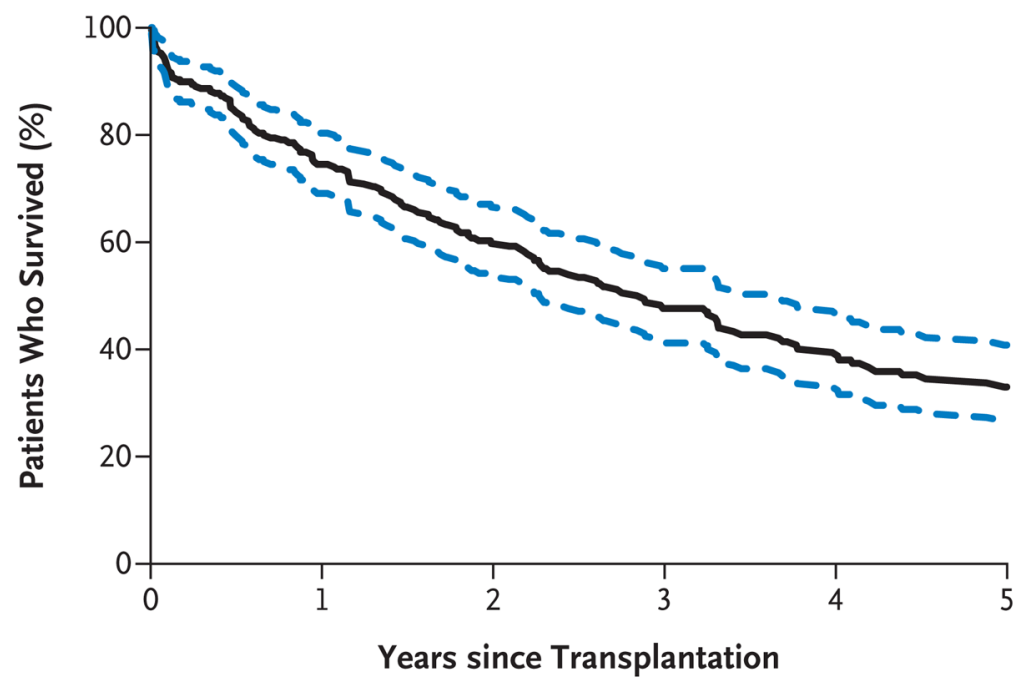

$\begin{array}{lllllll}\text { No. at Risk } & 248 & 164 & 117 & 83 & 59 & 44\end{array}$

Figure 1. Survival after Lung Transplantation among 248 Children with Cystic Fibrosis Who Were Younger than 18 Years of Age

The Kaplan-Meier curve (solid black line) is shown along with the upper and lower 95\% confidence limits (dashed blue lines). The median survival of these patients was 2.84 years (1037 days). The upper confidence limit shown here roughly matches the post-transplantation Kaplan-Meier survival curve in the report by Aurora et al. ${ }^{6}$ The rate of survival at 5 years was $32.9 \%$ - somewhat less than the $46.9 \%$ reported by the Organ Procurement and Transplantation Network for all recipients of lung transplants for cystic fibrosis. ${ }^{2}$ 


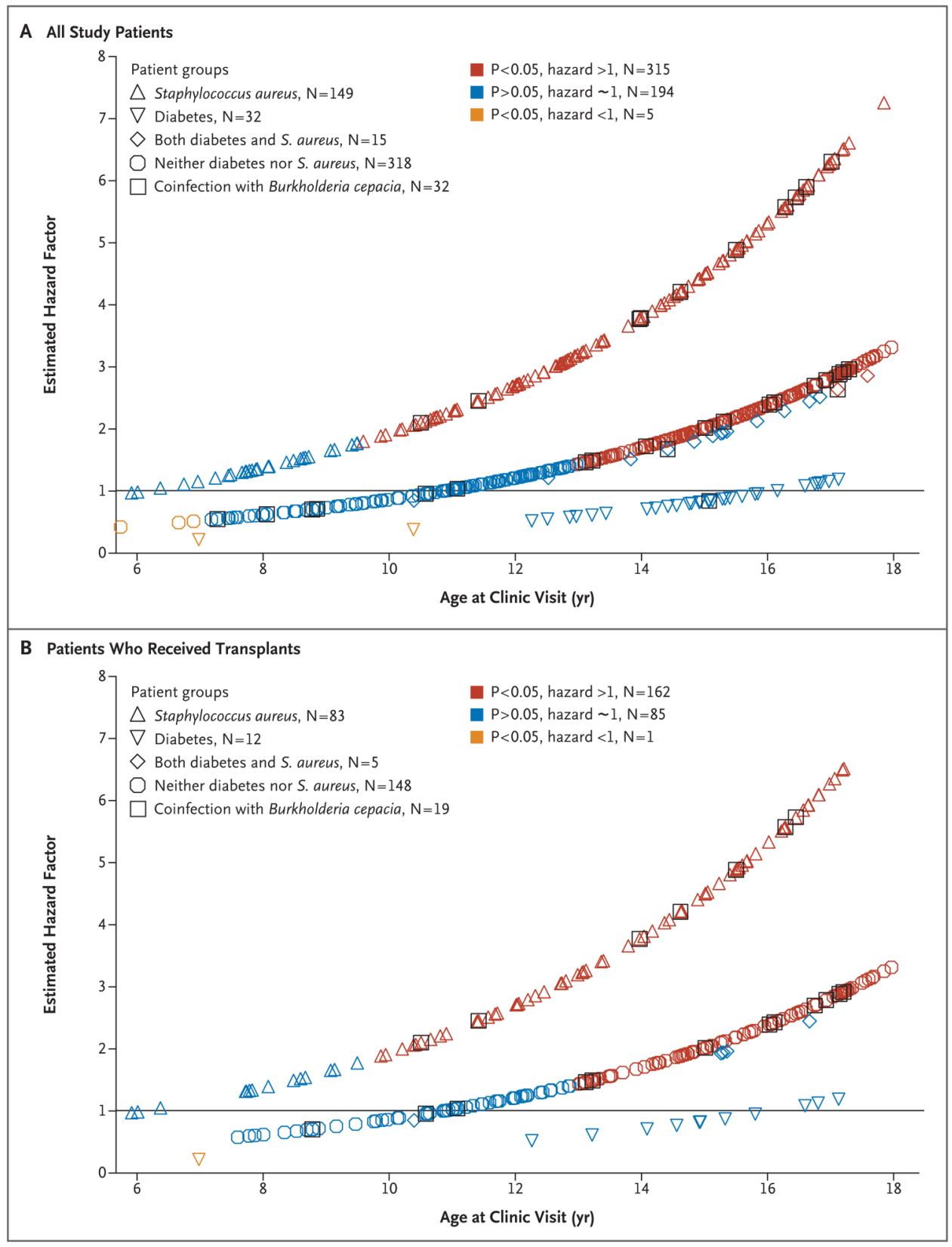

Figure 2. Estimated Hazard Factors Due to Lung Transplantation

Panel A shows the estimated hazard factors due to lung transplantation calculated for each of the 514 patients in the study, plotted as a function of age. Blue symbols denote the lack of power to determine a significant risk of harm or benefit from transplantation. Red symbols denote a significant risk of harm, and orange symbols a significant benefit. Patients are grouped according to four curves that correspond to the presence of either, both, or neither of the binary variables ( $S$. aureus infection and diabetes). The variable of B. cepacia infection does not interact with transplantation. The value of each hazard factor and the level of significance of each factor within each group at both extremes of age, where the data are sparse, should be viewed with caution, since they may have been the result of overextrapolation of the data. Unfortunately, all five of the patients with an apparently significant decrease in hazard factors (orange symbols) were included in this uncertain category. Panel B shows the estimated hazard 
factors due to lung transplantation for the 248 patients who underwent the procedure during the study period. These hazard factors are plotted as a function of age. Blue symbols denote the lack of power to determine a significant risk of harm or a significant benefit from transplantation. Red symbols denote a significant risk of harm, and orange symbols denote a significant benefit. A significant decrease in the hazard factor (orange symbol) is apparent for only one patient. 


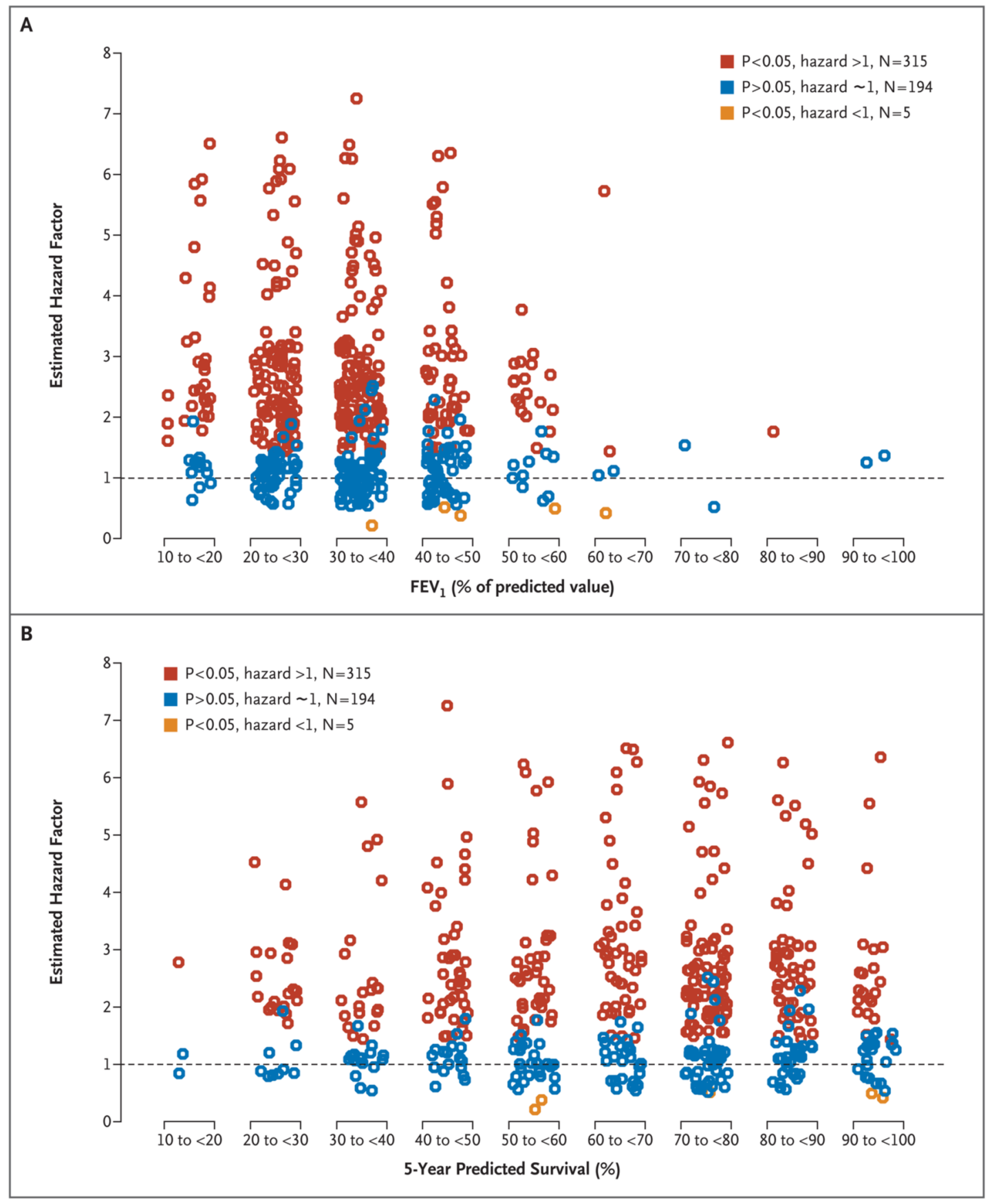

Figure 3. Estimated Hazard Factors for the 514 Patients in the Study According to Clinical Status Panel A shows the estimated hazard factors calculated as a function of the percent of the predicted value for forced expiratory volume in 1 second, but the data have been arranged as a series of nine scatter plots in order to identify strata. Panel B shows the estimated hazard factors as a function of the 5-year predicted survival probability, ${ }^{4}$ expressed as the percent likelihood of survival for 5 years after the date of the last clinic visit before placement on the waiting list. The data are arranged as a series of nine scatter plots in order to identify 5-year predicted survival strata. The hazard factor has no relationship with the 5-year predicted survival $(\mathrm{P}=0.87)$, but it decreases significantly with the $\mathrm{FEV}_{1} \%(\mathrm{P}<0.001)$. Patients with higher lung function appeared to have less harmful outcomes from lung transplantation, 
perhaps because of a significant decrease in the $\mathrm{FEV}_{1} \%$ with age. Neither $\mathrm{FEV}_{1} \%$ nor the 5year predicted survival probability is helpful for selecting patients who are likely to have a benefit from lung transplantation from among children with cystic fibrosis who have already been selected for the waiting list. 
Table 1

Characteristics of Patients at the Time of Placement on the Waiting List for Lung Transplantation. *

Variable

Age - yr
Median
Range

Female sex - \%

Deaths - no. $(\%)$

$\mathrm{FEV}_{1}-\%$ of predicted value ${ }^{\dagger}$

Acute exacerbations - no.

Mean

Range

Weight for age - z score

Pancreatic sufficiency - $\%$

Diabetes - \%

Burkholderia cepacia infection - \%

Staphylococcus aureus infection - \%

5 -yr predicted survival $-\% \$$

Mean

Range

* Plus-minus values are means \pm SD. Patients who did not receive transplants are those who did not receive a transplant before the end of the study.

$+$

${ }^{\dagger}$ The proportional-hazards method of analyzing survival corrects for significantly different covariates among study patients — in this case, for a significant difference in the forced expiratory volume in 1 second $\left(\mathrm{FEV}_{1}\right)$ between transplant recipients and patients who did not receive a transplant.

Fatients with diabetes were identified by the use of insulin.

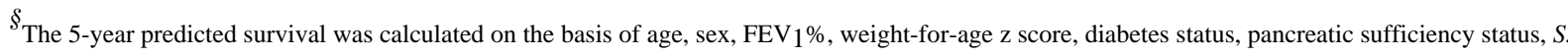
aureus infection and B. cepacia infection status, number of acute exacerbations of cystic fibrosis within 1 year, and an interaction term between $B$. cepacia and the number of acute exacerbations. The predicted survival was calculated from the date of the last clinic visit before the patient's placement on the waiting list for transplantation. $4,5,7$ 


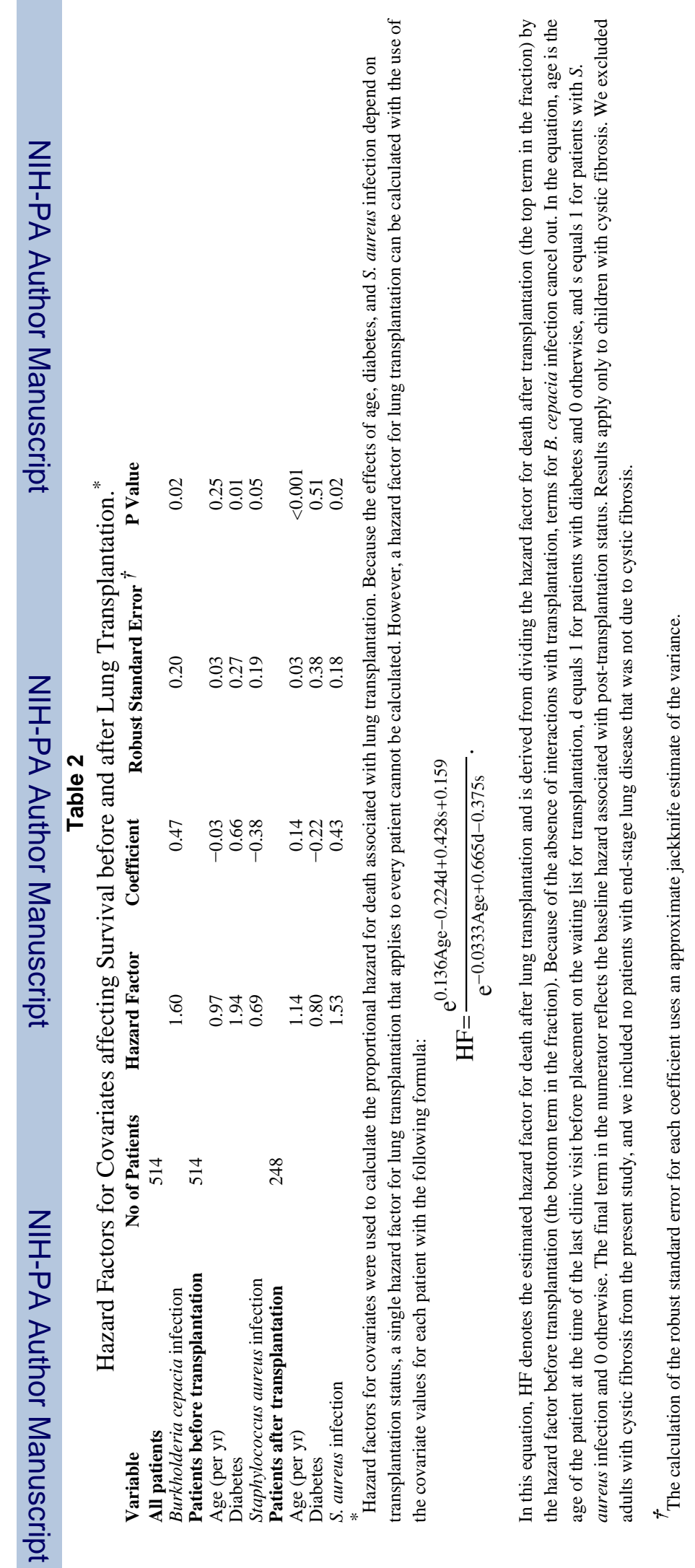

N Engl J Med. Author manuscript; available in PMC 2008 August 25. 